\title{
Subsymptomatic Aerobic Exercise for Patients With Postconcussion Syndrome: A Critically Appraised Topic
}

\author{
Katrina G. Ritter, Matthew J. Hussey, and Tamara C. Valovich McLeod
}

\begin{abstract}
Clinical Scenario: Patients who experience prolonged concussion symptoms can be diagnosed with postconcussion syndrome (PCS) when those symptoms persist longer than 4 weeks. Aerobic exercise protocols have been shown to be effective in improving physical and mental aspects of health. Emerging research suggests that aerobic exercise may be useful as a treatment for PCS, where exercise allows patients to feel less isolated and more active during the recovery process. Clinical Question: Is aerobic exercise more beneficial in reducing symptoms than current standard care in patients with prolonged symptoms or PCS lasting longer than 4 weeks? Summary of Key Findings: After a thorough literature search, 4 studies relevant to the clinical question were selected. Of the 4 studies, 1 study was a randomized control trial and 3 studies were case series. All 4 studies investigated aerobic exercise protocol as treatment for PCS. Three studies demonstrated a greater rate of symptom improvement from baseline assessment to follow-up after a controlled subsymptomatic aerobic exercise program. One study showed a decrease in symptoms in the aerobic exercise group compared with the full-body stretching group. Clinical Bottom Line: There is moderate evidence to support subsymptomatic aerobic exercise as a treatment of PCS; therefore, it should be considered as a clinical option for reducing PCS and prolonged concussion symptoms. A previously validated protocol, such as the Buffalo Concussion Treadmill test, Balke protocol, or rating of perceived exertion, as mentioned in this critically appraised topic, should be used to measure baseline values and treatment progression. Strength of Recommendation: Level C evidence exists that the aerobic exercise protocol is more effective than the current standard of care in treating PCS.
\end{abstract}

Keywords: rehabilitation, brain concussion, exercise training, return to play, persistent symptoms

\section{Clinical Scenario}

It is estimated that approximately 1.1 to 1.9 million sport-related concussions occur in patients 18 years or younger in the United States annually. ${ }^{1}$ Most adult patients who suffer from a concussion experience full relief from symptoms within 7 to 10 days of injury. ${ }^{2}$ However, approximately $10 \%$ to $20 \%$ of patients who sustain concussions experience symptoms that persist beyond this point. Patients who suffer from 3 or more symptoms, including headache, dizziness, fatigue, irritability, insomnia, concentration problems, or memory difficulty, for 4 or more weeks are diagnosed with postconcussion syndrome (PCS). ${ }^{3}$ Current recommendations for the treatment of concussions and PCS include physical and cognitive rest; however, this recommendation is vague, lacks evidence, ${ }^{4}$ and leaves a large degree of decision making up to the individual clinician. For a long time, it was believed that total rest until symptom resolution was the best practice for management of concussion and associated conditions. In recent years, there has been a shift in clinical treatment approaches that bring a more active recovery to the forefront of concussion management. ${ }^{5-8}$ These treatments vary based on the types of symptoms and deficits that persist after concussion.

Aerobic exercise protocols have been identified as a potentially beneficial treatment for individuals with persistent symptoms who are exacerbated by exertion. Prior research has used

The authors are with Athletic Training Program, A.T. Still University, Mesa, AZ. Ritter is also with Athletic Training Program, Department of Interdisciplinary Health Sciences, Arizona School of Heath Sciences, A.T. Still University, Mesa, AZ. Ritter (kritter@atsu.edu) is corresponding author. aerobic protocols to establish subsymptom baseline values. ${ }^{8}$ Patients are subjected to an initial aerobic protocol to establish the baseline symptomatic threshold. In the standard Balke Treadmill Concussion test, the baseline is determined by the patient reporting symptoms or unable to achieve full incline. ${ }^{5,9}$ In the studies included, there were minor variations in time and intensity utilized in the protocols. ${ }^{10-13}$ These values are then used to develop active rehabilitation protocols for patients after concussion in which they complete aerobic activity at a subsymptomatic threshold of between $60 \%$ and $80 \%$ of the baseline threshold. ${ }^{10-13}$

\section{Clinical Question}

Is aerobic exercise more beneficial in reducing symptoms than current standard care in patients with prolonged symptoms or PCS lasting longer than 4 weeks?

\section{Summary of Search, "Best Evidence" Appraised, and Key Findings}

- The literature was searched for studies that investigated whether an aerobic exercise protocol was more effective than the current standard of care for recovery from PCS.

- The literature search returned 17 possible studies related to the clinical question, of which 4 studies ${ }^{10-13}$ met the inclusion criteria and were included.

- The 4 studies ${ }^{10-13}$ included reported using aerobic exercise as a therapeutic intervention for PCS. 
- Participants who completed controlled subsymptomatic aerobic exercise experienced a greater rate of symptom improvement from baseline assessment for follow-up. ${ }^{10-13}$

- Subsymptomatic threshold can be determined through the Buffalo Concussion Treadmill test, Balke protocol, or Borg rating of perceived exertion (RPE). Patients with a concussion are not able to achieve maximum exertion. Symptom exacerbation occurs at a submaximum level. ${ }^{6,12-14}$

- Athletes responded to subsymptomatic threshold training faster than nonathletes. ${ }^{13}$

\section{Clinical Bottom Line}

There is moderate evidence to support subsymptomatic aerobic exercise as a treatment of PCS; therefore, it should be considered as a clinical option for reducing PCS and prolonged concussion symptoms. Clinicians should use a previously validated protocol to determine subsymptomatic threshold, including the Buffalo Concussion Treadmill test, Balke protocol, and Borg RPE. ${ }^{6,12-14}$ Although a specific timeline for duration of treatment has not been validated, there is evidence that 6 weeks of aerobic activity at $80 \%$ of subsymptom baseline may be effective in PCS treatment. ${ }^{10-13}$ Clinicians should utilize an individualized plan of care that includes patient baseline scores and personalized progression based on subsymptomatic threshold values.

\section{Strength of Recommendation}

Level $\mathrm{C}$ evidence exists that aerobic exercise protocol is more effective than the current standard of care in treating PCS.

\section{Search Strategy}

\section{Terms Used to Guide Search Strategy}

- Patient/Client group: participants with PCS or prolonged symptoms

- Intervention/Assessment: aerobic exercise

- Comparison: current standard care

- Outcome(s): symptom improvement or readiness to return to activity

- Time: concussion symptoms lasting longer than 4 weeks

\section{Sources of Evidence Searched}

- The Cochrane Library

- MEDLINE

- CINAHL

- SPORTDiscus

- Additional resources obtained via review of reference lists and hand search

\section{Inclusion and Exclusion Criteria}

\section{Inclusion Criteria}

- Studies that investigated patients diagnosed with concussion or sport-related concussion

- Studies that investigated patients with PCS or prolonged symptoms lasting longer than 4 weeks

- Studies that investigated aerobic exercise as a therapeutic intervention

- Limited to English language

- Limited to the past 10 years (2008-2017)

\section{Exclusion Criteria}

- Studies that investigated pharmacological interventions for treatment of PCS

- Studies that investigated subsymptomatic aerobic exercise as a tool for determining readiness to return to activity

\section{Results of Search}

Four relevant studies ${ }^{10-13}$ were located and categorized as shown in Table 1 (based on Levels of Evidence). ${ }^{15}$ Of the 4 studies identified, 3 studies are case series and 1 study is a randomized control trial.

\section{Best Evidence}

The studies in Table 2 were identified as the best evidence and selected for inclusion in this critically appraised topic. These studies were selected because they explored aerobic exercise as treatment for PCS.

\section{Implications for Practice, Education, and Future Research}

Several therapeutic interventions have been recommended for patients who have prolonged recovery after sustaining a concussion. ${ }^{8}$ This review proposed aerobic exercise as a comparison to the current standard of care and found that there is moderate evidence to support subsymptomatic aerobic exercise as a treatment of PCS. Because of current limitations in the literature, it is important that clinicians use sound clinical judgment when implementing this treatment. Three of the 4 studies included in this paper are case series that lack external validity due to their lack of a control group and limited generalizability to other populations. However, there is sound theoretical evidence that this treatment may help some patients with PCS. Despite the limitations of the available research, we believe that the consistent positive results warrant utilization of such a protocol for patients suffering from PCS.

Table 1 Summary of Study Designs of Articles Retrieved

\begin{tabular}{|c|c|c|c|}
\hline Level of evidence & Study design & Number located & References \\
\hline $1 \mathrm{~b}$ & Randomized control trial & 1 & Kurowski et al $^{12}$ \\
\hline 4 & Case series & 3 & Leddy et $\mathrm{al}^{13}{ }^{13}$ Gagnon et $\mathrm{al}^{11}$, and Chrisman et $\mathrm{al}^{10}$ \\
\hline
\end{tabular}




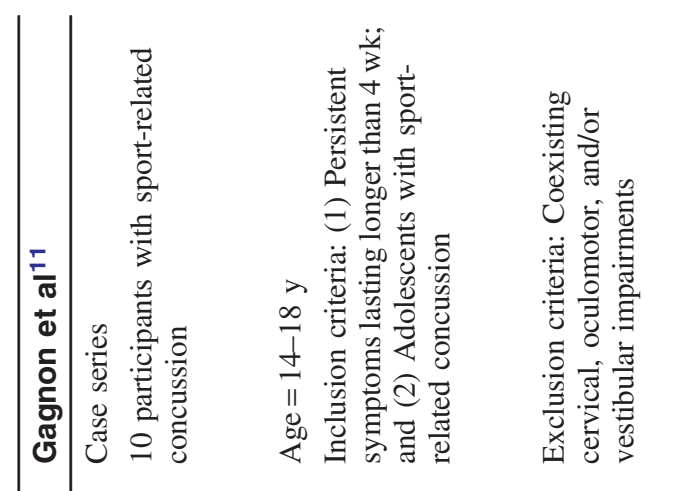

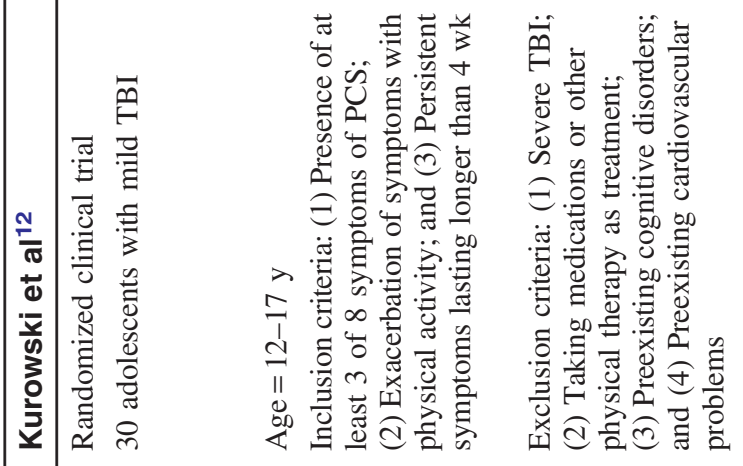

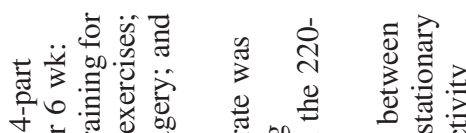

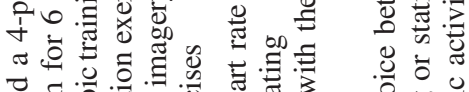

चี

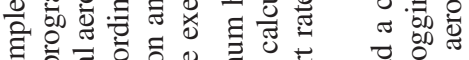

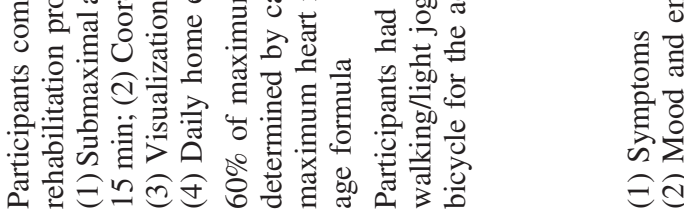

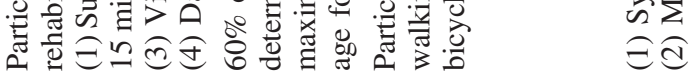

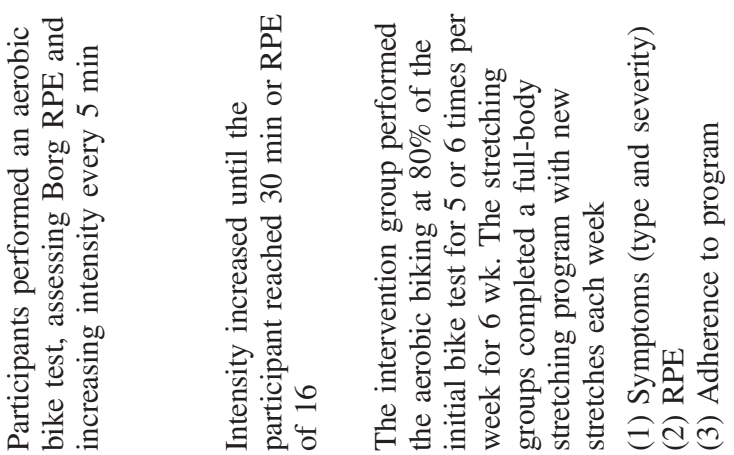

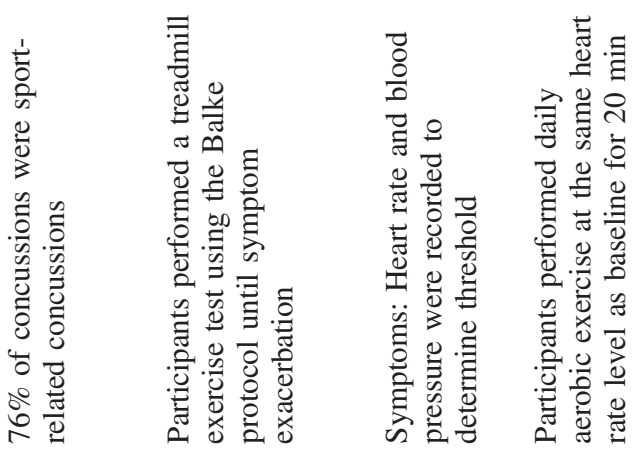

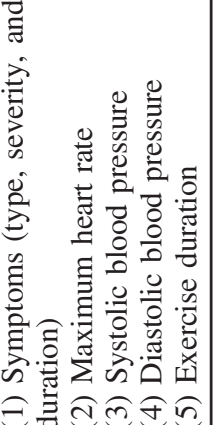

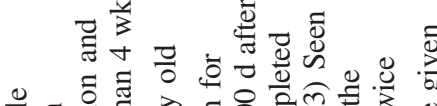

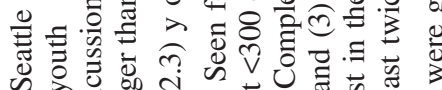

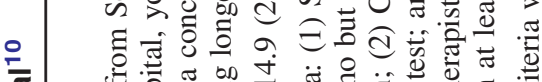

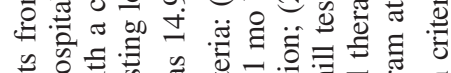

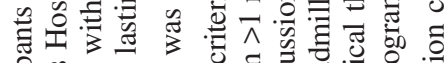

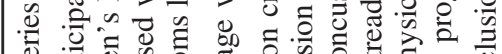

की

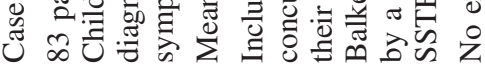
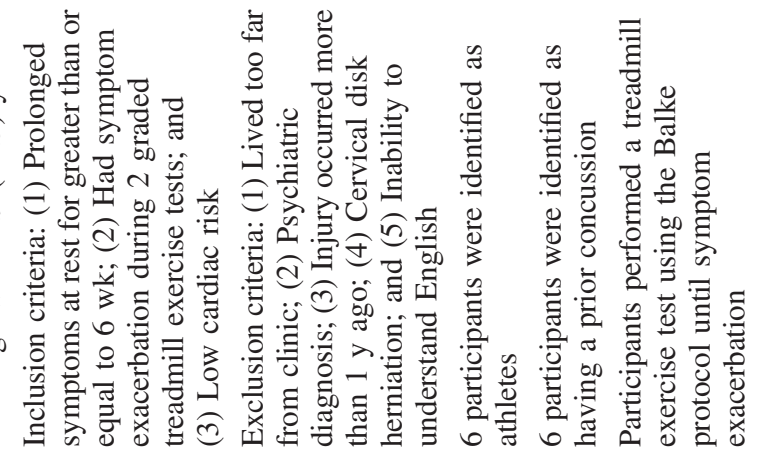

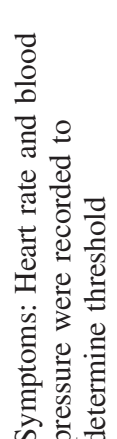

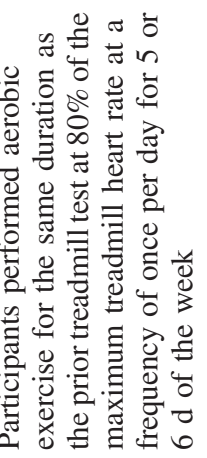

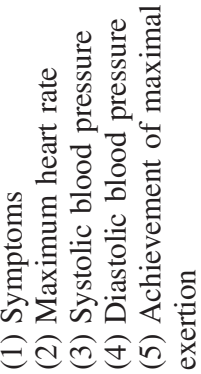




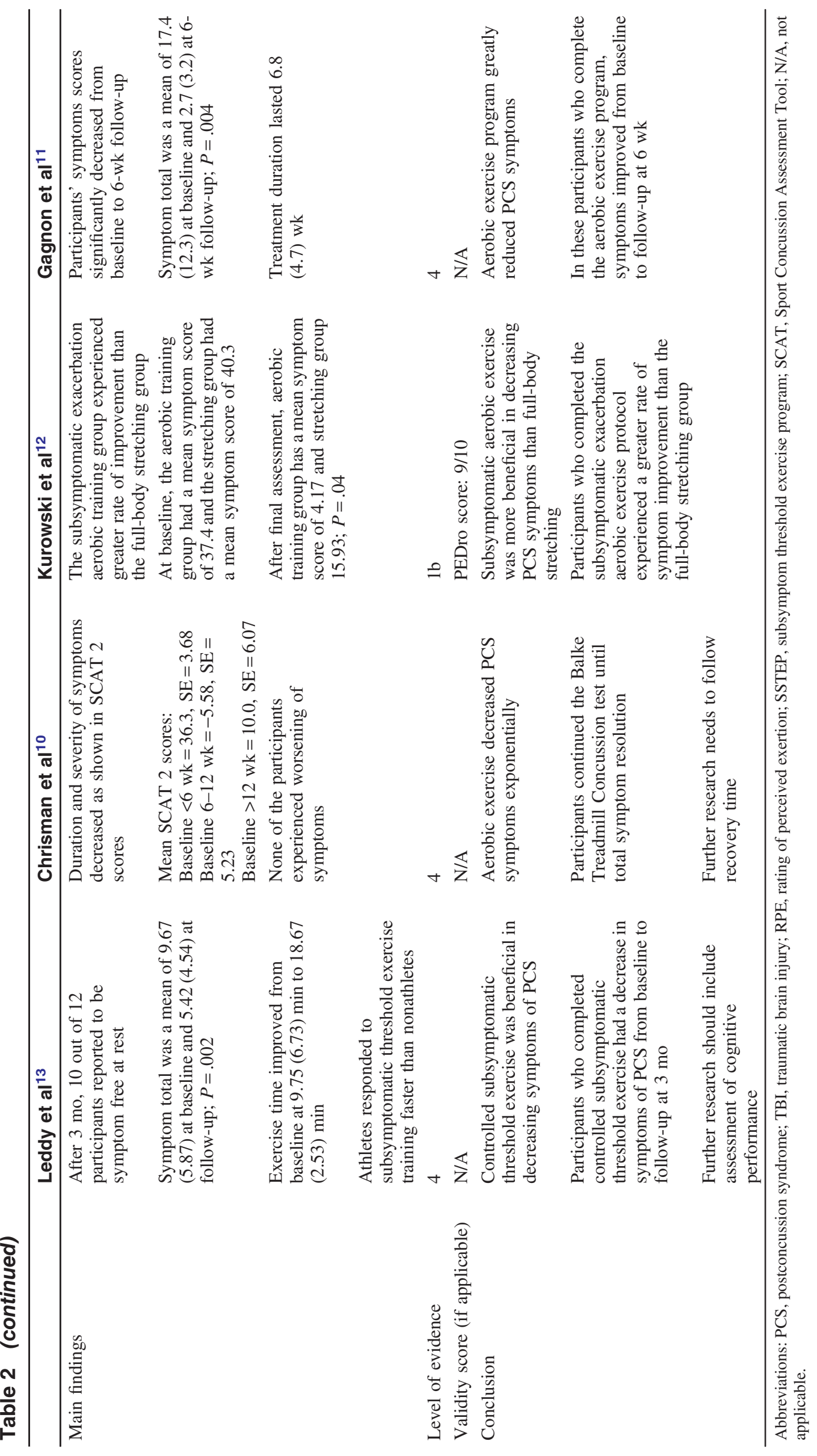


In patients who suffer from PCS and prolonged symptoms, 3 or more of the following symptoms are included: headache, dizziness, fatigue, irritability, insomnia, concentration problems, or memory difficulty; current recommendations suggest preliminary evidence is available to support the implementation of controlled subsymptomatic aerobic exercise. ${ }^{7,8}$ The subsymptomatic threshold should be determined using a validated measure like the Buffalo Concussion Treadmill test, Balke protocol, or Borg RPE. All 4 studies identified a treatment period of 6 to 9 weeks, with daily treatment at the subsymptomatic level that was predetermined through baseline testing. Three of the studies ${ }^{10,11,13}$ suggested daily aerobic exercise should last 15 to 20 minutes, and the other study ${ }^{12}$ required 30 minutes. By determining subsymptomatic threshold, patients can participate in subsymptomatic aerobic exercise to tolerance. This coincides with current concussion management recommendations, which focus on avoiding symptom exacerbation. ${ }^{7,16}$

Furthermore, there are emotional benefits of exercise as treatment of PCS. Exercise gives patients increased self-efficacy; less anxiety, stress, and depression; and better overall wellbeing. ${ }^{17-19}$ Regular exercise has shown to have cognitive benefits in improving cognitive function, sleep, learning, and memory. ${ }^{17,18}$ Athletes, who are conditioned to an active lifestyle, may be at risk of feeling isolated and removed during concussion recovery. An active approach to their rehabilitation might help improve overall cognitive function, decrease depression and isolation, and restore an active lifestyle that will help to promote healing. ${ }^{20}$ Leddy et al ${ }^{13}$ found that athletes responded to the aerobic exercise program faster than nonathletes, suggesting that aerobic exercise was beneficial specifically for athletes.

Although the current evidence for using submaximal exercise is promising, the level of evidence is low due to study design, number of participants, and population. However, a recent systematic review identified that it may be beneficial. ${ }^{8}$ Further research should include randomized controlled trials with large sample sizes and uniform control measures. Only 1 study identified the participants as having sport-related concussion, ${ }^{11}$ whereas 2 other studies identified and compared the participants' mechanism of injury as sport-related concussion or another mechanism. ${ }^{10,13}$ More research should be completed with a focus on individual populations, such as sport-related concussion, as there may be benefit of aerobic activity in those active prior to the injury. ${ }^{20}$

This critically appraised topic should be reviewed in 2 years, or when additional best evidence becomes available, to determine whether additional best evidence has been published that may change the clinical bottom line for the research question posed in this review.

\section{Acknowledgments}

There were no sources of funding to support this critically appraised topic. There are no conflicts of interest directly related to this critically appraised topic.

\section{References}

1. Bryan MA, Rowhani-Rahbar A, Comstock RD, Rivara F; Seattle Sports Concussion Research Collaborative. Sports- and recreationrelated concussions in US youth. Pediatrics. 2016;138(1):e20154635. PubMed ID: 27325635 doi:10.1542/peds.2015-4635

2. McCrory P, Meeuwisse WH, Aubry M, et al. Consensus statement on concussion in sport: the 4th international conference on concussion in sport held in Zurich, November 2012. Br J Sports Med. 2013;47(5): 250-258. PubMed ID: 23479479 doi:10.1136/bjsports-2013-092313

3. Boake C, McCauley SR, Levin HS, et al. Diagnostic criteria for postconcussional syndrome after mild to moderate traumatic brain injury. J Neuropsychiatry Clin Neurosci. 2005;17(3):350-356. PubMed ID: 16179657 doi:10.1176/jnp.17.3.350

4. Silverberg ND, Iverson GL. Is rest after concussion "the best medicine?": recommendations for activity resumption following concussion in athletes, civilians, and military service members. $J$ Head Trauma Rehabil. 2013;28(4):250-259. PubMed ID: 22688215 doi: 10.1097/HTR.0b013e31825ad658

5. Ellis MJ, Leddy J, Willer B. Multi-disciplinary management of athletes with post-concussion syndrome: an evolving pathophysiological approach. Front Neurol. 2016;7:136. PubMed ID: 27605923 doi:10.3389/fneur.2016.00136

6. Leddy JJ, Willer B. Use of graded exercise testing in concussion and return-to-activity management. Curr Sports Med Rep. 2013; 12(6):370-376. PubMed ID: 24225521 doi:10.1249/JSR.00000000 00000008

7. McCrory P, Meeuwisse W, Dvorak J, et al. Consensus statement on concussion in sport-the 5th international conference on concussion in sport held in Berlin, October 2016. Br J Sports Med. 2017; 51(11):838-847. PubMed ID: 28446457 doi:10.1136/bjsports2017-097699

8. Schneider KJ, Leddy JJ, Guskiewicz KM, et al. Rest and treatment/ rehabilitation following sport-related concussion: a systematic review. Br J Sports Med. 2017;51:930-934. PubMed ID: 28341726 doi:10.1136/bjsports-2016-097475

9. Darling SR, Leddy JJ, Baker JG, et al. Evaluation of the Zurich guidelines and exercise testing for return to play in adolescents following concussion. Clin J Sport Med. 2014;24(2):128-133. PubMed ID: 24184849 doi:10.1097/JSM.0000000000000026

10. Chrisman SP, Whitlock KB, Somers E, et al. Pilot study of the subsymptom threshold exercise program (SSTEP) for persistent concussion symptoms in youth. NeuroRehabilitation. 2017;40:493-499. PubMed ID: 28222566 doi:10.3233/NRE-161436

11. Gagnon I, Grilli L, Friedman D, Iverson GL. A pilot study of active rehabilitation for adolescents who are slow to recover from sportrelated concussion. Scand J Med Sci Sports. 2016;26(3):299-306. PubMed ID: 25735821 doi:10.1111/sms.12441

12. Kurowski BG, Hugentobler J, Quatman-Yates C, et al. Aerobic exercise for adolescents with prolonged symptoms after mild traumatic brain injury: an exploratory randomized clinical trial. J Head Trauma Rehabil. 2017;32(2):79-89. PubMed ID: 27120294 doi:10. 1097/HTR.0000000000000238

13. Leddy JJ, Kozlowski K, Donnelly JP, Pendergast DR, Epstein LH, Willer B. A preliminary study of subsymptom threshold exercise training for refractory post-concussion syndrome. Clin J Sport Med. 2010;20(1):21-27. PubMed ID: 20051730 doi:10.1097/JSM. 0b013e3181c6c22c

14. Leddy JJ, Baker JG, Kozlowski K, Bisson L, Willer B. Reliability of a graded exercise test for assessing recovery from concussion. Clin J Sport Med. 2011;21(2):89-94. PubMed ID: 21358497 doi: 10.1097/JSM.0b013e3181fdc721

15. OCEBM Levels of Evidence Working Group. The Oxford 2011 Levels of Evidence. Oxford Centre for Evidence-Based Medicine. 2011. http://www.cebm.net/index.aspx?o=5653

16. McLeod TCV, Gioia GA. Cognitive rest: the often neglected aspect of concussion management. Athl Ther Today. 2010;15(2):1-3. doi:10. 1123/att.15.2.1

17. Hogan CL, Catalino LI, Mata J, Fredrickson BL. Beyond emotional benefits: physical activity and sedentary behaviour affect psychosocial 
resources through emotions. Psychol Health. 2015;30(3):354-369. PubMed ID: 25307453 doi:10.1080/08870446.2014.973410

18. McKee AC, Daneshvar DH, Alvarez VE, Stein TD. The neuropathology of sport. Acta Neuropathol. 2014;127(1):29-51. PubMed ID: 24366527 doi:10.1007/s00401-013-1230-6

19. Rasmussen L, Justice D, Chang KW, Nelson VS, Yang LJ. Home exercise DVD promotes exercise accuracy by caregivers of children and adolescents with brachial plexus palsy. $P M$ R. 2013;5(11): 924-930. PubMed ID: 23770351 doi:10.1016/j.pmrj.2013.06.003

20. Mychasiuk R, Hehar H, Ma I, Candy S, Esser MJ. Reducing the time interval between concussion and voluntary exercise restores motor impairment, short-term memory, and alterations to gene expression. Eur J Neurosci. 2016;44(7):2407-2417. PubMed ID: 27521273 doi:10.1111/ejn.13360 